\title{
ORIGINAL ARTICLE \\ The effects of resource availability and the demographic transition on the genetic correlation between number of children and grandchildren in humans
}

\author{
E Bolund ${ }^{1}$ and V Lummaa ${ }^{2}$ \\ Studies of evolutionary change require an estimate of fitness, and lifetime reproductive success is widely used for this purpose. \\ However, many species face a trade-off between the number and quality of offspring and in such cases number of grandoffspring \\ may better represent the genetic contribution to future generations. Here, we apply quantitative genetic methods to a \\ genealogical data set on humans from Finland to address how the genetic correlation between number of children and \\ grandchildren is influenced by the severity of the trade-off between offspring quality and quantity, as estimated by different \\ levels of resource access among individuals in the population. Further, we compare the genetic correlation before and after the \\ demographic transition to low mortality and fertility rates. The genetic correlation was consistently high (0.79-0.92) with the \\ strongest correlations occurring in individuals with higher access to resources and before the demographic transition, and a \\ tendency for lower correlations in resource poor individuals and after the transition. These results indicate that number of \\ grandoffspring is a slightly better predictor of long-term genetic fitness than number of offspring in a human population across a \\ range of environmental conditions, and more generally, that patterns of resource availability need to be taken into account when \\ estimating genetic covariances with fitness.
}

Heredity (2017) 118, 186-192; doi:10.1038/hdy.2016.81; published online 14 September 2016

\section{INTRODUCTION}

How to best define and measure fitness is a long-standing issue in ecology and evolutionary biology (Stearns, 1976; Endler, 1986; McGraw and Caswell, 1996; Wolf and Wade, 2001; Brommer et al., 2004). The most accurate estimates of fitness would compute the number of descendants an infinite number of generations into the future (Hunt et al., 2004), but empirical estimation of this is intractable because there is no single parameter that is universally suitable (De Jong, 1994). Therefore, empiricists across taxa (Brommer et al., 2004; Hunt et al., 2004; Stearns et al., 2010) need to rely on measurable proxies for fitness, such as metrics of reproductive success, like number of offspring born (lifetime reproductive success, LRS), with the assumption that this will reflect long-term genetic contribution to the population.

However, a fundamental idea in evolutionary biology is a trade-off between the number of offspring produced and their 'quality' (Lack, 1947; Roff, 2002). This builds on the assumptions that (1) resources are limited such that per offspring investment will decrease as number of offspring increases, and (2) the future lifetime reproductive success of each offspring is increased with increased parental investment. Thus, number of offspring may not be an accurate prediction of an individual's genetic contribution to future generations. The magnitude of the trade-off between fertility (that is, number of offspring produced) and offspring quality varies and depends on factors such as extent of parental care: the trade-off will be especially pronounced in animals with protracted periods of parental care, and experimental studies in avian and mammalian species have convincingly shown that increased fertility has negative effects on offspring survival (Roff, 2002). Although studies on humans have found mixed results, a recent review concluded that there is good reason to believe that a trade-off between quantity and quality of offspring is fundamental to human life history (Lawson and Mace, 2011). High infant and childhood mortality in traditional societies meant that half, or fewer, of born individuals actually contributed to future generations during human evolution (Wells and Stock, 2007), thus arguing that number of grandchildren may be a better estimate of fitness than number of children in humans, because it combines offspring production, recruitment and subsequent fertility into an aggregated longer-term estimate of individual fitness. In contrast, a recent study on a post-industrial human population that had gone through the demographic transition in Sweden (using the Uppsala multigenerational Birth Cohort Study) found that number of children strongly predicted long-term fitness measured over four generations (Goodman et al., 2012), arguing for the use of LRS to estimate current selective pressures in post-demographic transition populations. However, although the same study found that high fertility did not compromise the survival, mating success or reproductive success of grandchildren or great-grandchildren, reproductive behaviours that promoted long-term genetic fitness did not promote descendant socioeconomic success.

Thus, one explanation for conflicting results regarding the presence of a quality/quantity trade-off in humans may be the differences in

${ }^{1}$ Evolutionary Biology Centre, Department of Ecology and Genetics, Uppsala University, Uppsala, Sweden and 2Department of Biology, University of Turku, Turku, Finland Correspondence: Dr E Bolund, Evolutionary Biology Centre, Department of Ecology and Genetics, Uppsala University, Norbyvägen 18D, SE-752 36 Uppsala, Sweden. E-mail: elisabeth.bolund@ebc.uu.se

Received 4 February 2016; revised 29 July 2016; accepted 1 August 2016; published online 14 September 2016 
resource limitation between the studied populations, because such trade-offs may be observable only when resources are limited (van Noordwijk and Jong, 1986; Meij et al., 2009). However, in resource limited settings mortality can be mainly extrinsic, making trade-offs difficult to detect in correlational studies. Although most individuals in post-industrial populations in general have access to relatively abundant resources, pre-industrial populations can suffer from strong resource limitation. For example, in historical Finland, poor crop yields resulted in frequent famines, which were especially severe for individuals of low socioeconomic status (SES; Hayward et al., 2012). High birth and death rates before industrialisation likely promote high variability in the quality of individual children. These conditions are thus likely to closer reflect the conditions that have characterised much of our evolutionary history (Wells and Stock, 2007). Generally coinciding with industrialisation, countries went through a period of steeply decreasing mortality and fertility rates: the demographic transition (Borgerhoff Mulder, 1998). Thus, results on post-industrialised populations that have gone through the demographic transition may not generalise to pre-industrial populations.

Another reason for the lack of consistent findings may be a persistent focus on the phenotypic level in studies of human life history evolution (Lummaa, 2013). However, for evolution (that is, the change in allele frequencies in the population over time) it is the longterm genetic fitness that is important (Roff, 2002). Hence, although phenotypic measures, such as number of children or grandchildren, aim to estimate the genetic quality of individuals (Hunt et al., 2004) it would be more fruitful to directly estimate the genetic variance in these traits. A recent study used the classical twin design on a data set from post-industrial Sweden (the Swedish Twin Registry) and found that number of children and grandchildren were perfectly genetically correlated (that is, that $r_{g}$ was not significantly different from 1 , Zietsch et al., 2014). A genetic correlation of one indicates that the genetic basis of the two traits is completely overlapping, and thus both measures perform equally well as fitness proxies. A genetic correlation lower than one indicates a partly separate genetic basis of the two traits, meaning that number of grandchildren is a closer proxy of fitness. Thus, the genetic variation in number of children completely overlapped with that in number of grandchildren and there was very little independent genetic variance in the two traits. This has important implications for studies of evolutionary change, because projections of evolutionary change in traits depends on the covariances between traits and fitness (Falconer and Mackay, 1996). The study suggested that LRS may be a sufficient proxy for fitness in modern human populations. However, as pointed out above, conditions were very different before the demographic transition and it is therefore imperative to find out if number of children and grandchildren are also similarly genetically correlated in such populations. Historical data sets largely preclude the use of twins, because historical records rarely distinguish between mono- and dizygotic twins. Instead, methods that make use of information from relatives in several successive generations can be used to estimate quantitative genetic parameters (Bolund et al., 2016).

Here, we investigate the effect of resource limitation and the demographic transition on the genetic correlation between number of children and grandchildren. We use data collected from church records in Finland from the last three centuries which allows us to form pedigrees over multiple generations. The demographic transition in Finland happened relatively late compared with other European countries, approximately from the 1880s onwards (Singleton, 1998; Bolund et al., 2015), allowing comparisons before and after the demographic transition. Further, occupational data allows us to assign individuals to socioeconomic classes, which reflect variation in resource access. We can therefore compare subsets of the population with different levels of resource limitation. The data also allows controlling statistically for twinning status, birth order and place of birth. Taken together, this offers a rare opportunity to simultaneously assess the role of resource access and changing fertility and mortality patterns on both the phenotypic, and importantly, the genetic correlation between number of children and grandchildren and determine whether LRS is a sufficient proxy for fitness in a human population under varying environmental conditions.

\section{MATERIALS AND METHODS}

\section{Data}

Our data come from Finnish church records from 1705 to 2011 from five inland (Ikaalinen, Jaakkima, Pulkkila, Rautu and Tyrvää) and three coastal parishes (Hiittinen, Kustavi and Rymättylä). The Lutheran church was obliged by law to accurately record all births, marriages and deaths in every parish in the country since the 18th century. We use these data to construct social pedigrees for a large number of individuals. We included individuals that had complete life history data from two consecutive generations, meaning that the individual and all of his/her offspring (if he/she reproduced) were followed at least until the age of 45 for women and 50 for men to allow a full potential reproductive lifespan. This allows the accurate measurement of number of grandchildren born and resulted in a representative sample of individuals living both before and after the demographic transition (Table 1). For quantitative genetic analyses, the full pedigree (75 223 individuals) was pruned to include only individuals with full information for all fixed and random effects that were included in the analyses (see below). This resulted in 8342 individuals with 2667 mothers, 2583 fathers, 2581 full siblings and a maximum pedigree depth of 6 generations in the full dataset used for analyses. Mean maternal sibship size was 1.60 and mean paternal sibship size was 1.62 . Our estimates are unlikely to be biased by extra-pair paternity because strict laws against adultery were enforced during most of the study period (Sundin, 1992). Levels likely ranged between 1.7 and $3.3 \%$ as has also been suggested for modern populations with high paternity confidence (Anderson, 2006), and which has been confirmed to be the case for other similar church record based pedigrees from historical Europe (Larmuseau et al., 2013). Such low levels of extra-pair paternity are insufficient to qualitatively bias quantitative genetic estimates (Charmantier and Réale, 2005), even if pedigree errors are non-random with respect to male traits (Firth et al., 2015). Given this robustness to moderate levels of pedigree errors, it is likely that quantitative genetic estimates can be generalised across populations with modest variation in levels of extra-pair paternity.

\section{Resource availability}

To contrast our findings in individuals with different access to wealth, we used occupational data to divide individuals into two SES. Before the demographic transition, these statuses refer to landowners who owned land and landless who

Table 1 Phenotypic means ( \pm s.d.) for number of children and grandchildren born

\begin{tabular}{lcccc}
\hline Subset & $\begin{array}{c}\text { Mean }( \pm \text { s.d.) } \\
\text { children }\end{array}$ & $\begin{array}{c}\text { Mean }( \pm \text { s.d.) } \\
\text { grandchildren }\end{array}$ & $\begin{array}{c}\mathrm{N} \\
\text { total }\end{array}$ & $\begin{array}{c}\mathrm{N} \\
\text { reproducing }\end{array}$ \\
\hline Pre 1880 & $2.40 \pm 2.93$ & $5.20 \pm 8.92$ & 3246 & 1964 \\
Post 1880 & $1.47 \pm 2.17$ & $2.11 \pm 4.04$ & 1005 & 474 \\
Landowner & $3.05 \pm 3.10$ & $6.94 \pm 9.93$ & 2238 & 1608 \\
Landless & $0.97 \pm 1.84$ & $1.34 \pm 3.93$ & 1008 & 356 \\
\hline
\end{tabular}

The table is based on the full sample including both reproducing and non-reproducing individuals, shown separately for two time periods before versus after the demographic transition. Within the pre-demographic transition time period, estimates are also shown separately for two socioeconomic classes, landowner and landless. Sample sizes $(N)$ are given for the full sample and for the subset that reproduced. 
either rented land or had no access to land at all (Pettay et al., 2007). After the demographic transition and concomitant industrialisation, the SES classes refer to economically rich versus poor individuals. Women who did not have a profession were assigned the SES of the (first) husband with which they reproduced. Although this dichotomous assignment of SES is based on occupation data only (in contrast to the inclusion of, for example, education, which however, was not available in the studied population during most of the study period), these two categories have consistently been associated with variation in many aspects of fitness in previous studies on this population (Pettay et al., 2007; Gillespie et al., 2008; Hayward et al., 2012; Liu and Lummaa, 2014). Furthermore, broad categories are necessary to achieve sufficient sample sizes within each SES group for quantitative genetic analyses. Before the demographic transition, landless individuals suffered more from frequent famines and epidemics of infectious diseases in this population, while landowners were protected from these negative extremes of fluctuations in resource availability (Hayward et al., 2012). In general, SES has been shown to influence long-term fitness (Pettay et al., 2007) and life history trade-offs (Gillespie et al., 2008; Liu and Lummaa, 2014) in this, as well as other preindustrial populations (Voland, 1990; Skjærvø et al., 2011). After industrialisation, these differences between socioeconomic classes with respect to resource availability diminished, which is reflected in smaller differences in reproductive success between poor and rich individuals (Liu and Lummaa, 2014, Results section). Therefore, we here focus on individuals born before 1880 when investigating the effect of resource availability on the relationship between number of children and grandchildren. Individuals showed fairly high intergenerational mobility between socioeconomic classes: $67 \%$ of women and $70 \%$ of men born between 1705 and 1880 belonged to the same socioeconomic class as their social father.

\section{Phenotypic analyses}

To estimate the phenotypic correlation between number of children and grandchildren in the different subsets, we used generalised linear mixed-effects models with penalised quasi likelihood (glmmPQL). Models were run in R in the packages nlme and MASS (Venables and Ripley, 2002; Pinheiro et al., 2008). Number of children and grandchildren were $\mathrm{z}$-standardised (for each value within each subset, the mean value of the trait was subtracted and the result divided by the s.d. of the trait) so that the slope estimates can be interpreted as standardised partial regression coefficients (Schielzeth, 2010). This allows us to estimate the effect of number of children on number of grandchildren, while statistically holding the effects of a range of fixed and random effects constant. A quadratic effect of number of children was initially added in each model to estimate curvature of the relationship, and this was removed in the final models if non-significant. As fixed effects, we included the individual's own twinning status at birth as a two-level factor (singleton or multiple) because it affects fitness (Lummaa et al., 1998), birth order as a two-level factor (firstborn son versus others) because the firstborn son inherited the majority of the wealth (Faurie et al., 2009), parish of origin as a factor with eight levels and sex of the individual as a two-level factor (Bolund et al., 2013). In models that focussed on estimating parameters in separate time periods, SES was included as a two-level fixed effect. Finally, birth cohort (divided into 20-year intervals) and maternal identity were added as random effects to control for heterogeneity within time periods and due to family structure. Because these models account for the uncertainty in the response variable only, we also estimated phenotypic correlations from the animal models (described in more detail below). These correlations account for variance due to fixed and random effects for both number of children and number of grandchildren. However, because of the added computational complexity that is required to estimate non-linear effects in the animal models, we restricted these models to the estimation of linear effects. Nonetheless, results from both methods were highly consistent.

\section{Quantitative genetic analyses}

The 'animal model' provides a powerful means to use all the information available in complex, natural pedigrees to estimate additive genetic variances and covariances whilst controlling for confounding factors, and has thus been very widely applied in recent decades in evolutionary biology (Henderson, 1975; Kruuk, 2004; Wilson et al., 2010). We applied animal models with a
Bayesian Monte Carlo Markov chain mixed-effects modelling approach implemented in the R-package MCMCglmm (Hadfield, 2010). MCMCglmm provides meaningful error estimates for derived variables by direct sampling from the posterior distribution. Although the demographic transition clearly was a gradual process, it remains a methodological challenge to study changes in quantitative genetic parameters over a continuous scale. We therefore chose a simplified analytic approach, and used the year 1880 to divide our data into pre- and a post-demographic transition subsets. Thus, we ran four bivariate models, one for each time period and one for each socioeconomic class in the time period before 1880 to estimate a separate additive genetic variancecovariance matrix, G, for each subset. Each model fitted number of children and grandchildren born as response variables:

$$
\begin{aligned}
& \left(\text { Trait1, trait2) } \sim[\mathrm{SES}]+\text { Twin }+ \text { Firstbornson }+ \text { Parish }+ \text { Sex }+V_{\mathrm{A}}\right. \\
& \quad+V_{\text {BirthC }}+V_{\mathrm{M}}+V_{\mathrm{R}}
\end{aligned}
$$

where $V_{\mathrm{A}}$ is the additive genetic effect, $V_{\mathrm{BirthC}}$ is the random effect due to birth cohort, $V_{\mathrm{M}}$ is the random effect due to maternal identity, $V_{\mathrm{R}}$ is the residual (or environmental) variance and SES was added as a fixed effect in the two models that focussed on time effects. The variances and covariances are estimated after removing the variation due to the fixed effects. From these models, we could estimate not only the variance in each trait accounted for by additive genetic, maternal, birth cohort and residual effects but also the additive genetic and the phenotypic covariance (and hence correlation) between the two traits. We calculated the heritability $\left(h^{2}\right)$ along with the $95 \%$ Bayesian credibility interval of each trait from the posterior distribution of variance component estimates as $h^{2}=V_{\mathrm{A}} / V_{\mathrm{P}}$. Because the phenotypic variances were different between the two traits in the subgroups (Table 1), traits were z-standardised within subsets prior to analyses when calculating standardised estimates $\left(h^{2}\right.$ and genetic correlations). To allow interpretation on the scale of measurement, $V_{\mathrm{A}}$ and $V_{\mathrm{R}}$ were estimated in models using mean-standardised trait values.

We specified a weakly informative invese-Gamma prior (specifying a $\mathrm{V}$ of 2 and an $n$ of 1.002). Estimates were robust to the use of an alternative weakly informative prior that partitions the phenotypic variance of the traits of interest evenly among each random effect (and covariances to zero) and specifying a degree of belief parameter $(n)$ equal to the size of the matrix. Convergence of runs was confirmed by visual inspection of output plots and by assuring that autocorrelation between consecutive samples did not exceed 0.1 (Hadfield, 2010). Convergence was achieved with runs of 200000 to 1000000 iterations (after an initial burn-in of 10000 iterations), and every 200-1000th iteration sampled for a total of 1000 samples from the posterior distribution.

\section{RESULTS}

\section{Demographic parameters}

During the demographic transition, levels of child mortality and fertility decreased dramatically. For example, among individuals with landowning fathers, $58 \%$ of those born in the 1860 s survived to adulthood ( $N=625$ individuals with known SES of father) while $47 \%$ of individuals with landless fathers survived $(N=2945)$. In comparison, survival to adulthood was $92 \%$ for both SES classes during the 1940s ( $N=1082$ landowning versus 252 landless). During the same decades lifetime reproductive success among reproducing individuals decreased from $4.9( \pm 3.1)$ children for landowning individuals born in the 1860s $(N=1301$ individuals with known SES $)$ and $3.8( \pm 2.8)$ in landless individuals $(N=627)$ to $1.8( \pm 1.2, N=625)$ in landowning versus $1.3( \pm 1.2, N=176)$ in landless for those born in the 1940 s.

\section{Phenotypic correlations}

As expected, standardised partial regression coefficients between the number of children and grandchildren were consistently high and did not differ significantly between the four subsets, indicating that high numbers of children consistently translated to high numbers of grandchildren (Figure 1). Estimates were slightly lower in landless (linear standardised estimate \pm s.e.: $0.68 \pm 0.024, t=27.8, N=1008$ ) than in landowning individuals $(0.70 \pm 0.015, t=48.3, N=2238)$. 
a

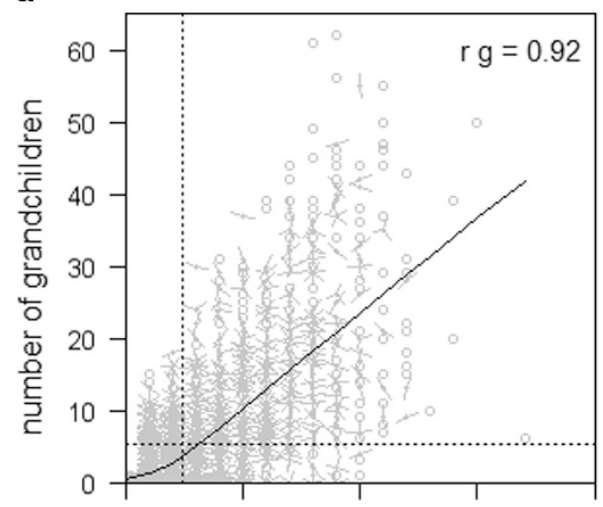

C

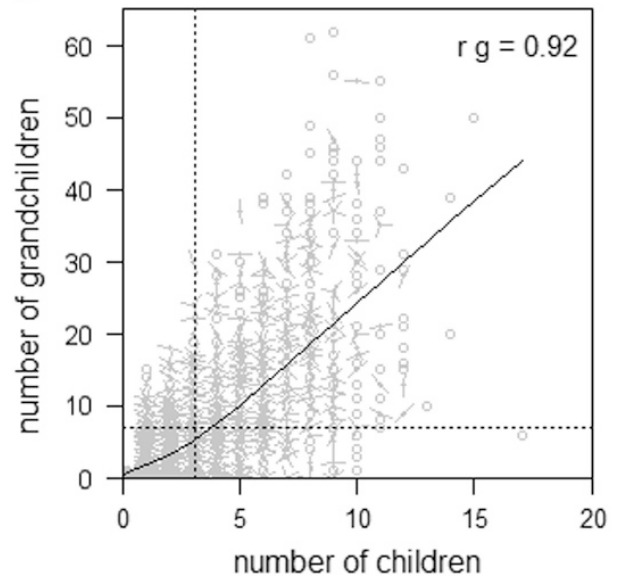

b

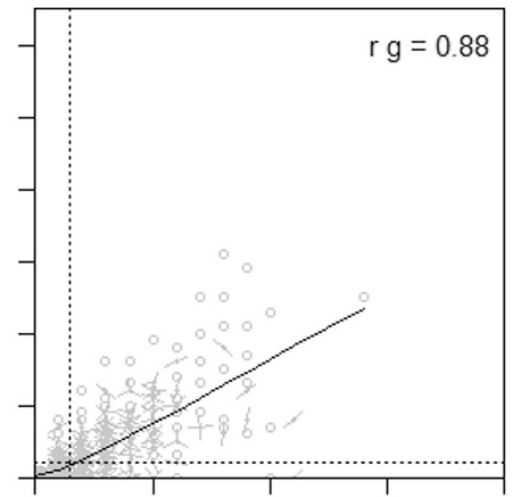

d

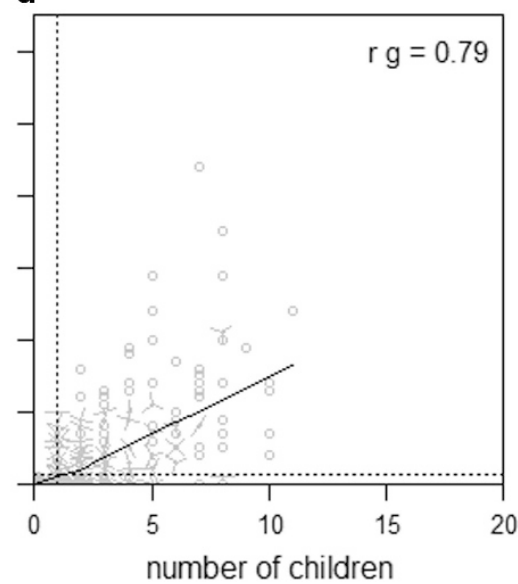

Figure 1 The phenotypic relationship on the scale of measurement between number of children and grandchildren born, illustrated separately for (a) all individuals born before 1880 and (b) all individuals born after 1880. Within the pre-demographic transition subset, (c) shows landowning individuals and (d) shows landless individuals. The number of observations in each point is illustrated by the number of lines in the sunflower point. Full black lines represent locally weighted smoothing curves based on the predicted values from gImmPQL models that control for a number of fixed and random effects in the response variable. Dotted black lines show the mean number of children (vertical line) and grandchildren (horizontal line) in each subset, including both reproducing and non-reproducing individuals. The label in each graph indicate the genetic correlation $(r \mathrm{~g})$ between number of children and grandchildren, as estimated in separate models (for details, see text).

Estimates were slightly higher after the demographic transition (before 1880: $0.72 \pm 0.012, t=60.8, N=3246$ versus after $1880: 0.74 \pm 0.022$, $t=34.1, N=1005)$. The relationship between number of children and grandchildren was very weakly quadratic in landless individuals indicating diminished gains of grandchildren from very high numbers of born children (model with second degree polynomial term: quadratic estimate: $0.041 \pm 0.016, t=2.59$, linear estimate: $0.58 \pm 0.047, t=12.2$ ) and there was a trend for a U-shaped relationship after 1880 (model with second degree polynomial term: quadratic estimate: $-0.025 \pm 0.013, t=1.90$, linear estimate: $0.79 \pm 0.035, t=22.7$ ).

Thus, an increase of one population s.d. in number of children increased the number of grandchildren by 0.68 population s.d.'s in landless and 0.70 in landowning individuals born before 1880. This translates into an increase in 2.1 grandchildren for each additional child born in landless individuals and 3.2 grandchildren for each additional child in landowning individuals. Phenotypic correlations estimated from animal models were consistent with these results (Supplementary Table 1).

\section{Genetic and environmental variances}

Both the additive genetic and the environmental variances of number of children and grandchildren were markedly higher in landowning than landless individuals and higher before the transition compared with after (Table 2, note that the $95 \%$ credibility intervals are not overlapping). The ratio of $V_{\mathrm{A}}$ to the total variance (the $h^{2}$ ) tended to show a similar pattern for number of children. Thus, a higher proportion of the variation in number of children were explained by genetic differences between individuals in landowning as compared to landless individuals, as well as before as compared to after the demographic transition. The heritability of grandchildren remained largely stable because both the $V_{\mathrm{A}}$ and $V_{\mathrm{E}}$ changed proportionally in the same directions, resulting in a stable ratio (Table 2). Maternal effects were consistently low, explaining $11 \%$ of the total variance in landless individuals before industrialisation, and $3 \%$ or less of the total variance in all other subsets.

\section{Genetic correlations}

We found a genetic correlation between number of children and grandchildren that was consistently $<1$ in both time periods and socioeconomic classes, indicating that while there was a large overlap in the variance in the genetic basis of number of children and grandchildren born, the two are not perfectly correlated. The genetic correlation tended to be slightly lower after the demographic transition (0.88, CI: 0.74-0.94) compared with before the transition (0.92, CI: 0.88-0.96). Further, before the transition, the genetic correlation 
Table 2 Quantitative genetic parameters for two fitness measures

\begin{tabular}{lcccc}
\hline & Pre 1880 & Post 1880 & Landowner \\
\hline Heritability children & $0.16(0.11-0.21)$ & $0.092(0.040-0.17)$ & $0.19(0.13-0.25)$ & $0.15(0.10-0.21)$ \\
Heritability grandchildren & $0.15(0.096-0.19)$ & $0.16(0.069-0.25)$ & $1.82(1.22-2.54)$ & $0.14(0.038-0.32)$ \\
$V_{\text {A children }}$ & $1.25(0.94-1.77)$ & $0.51(0.27-0.81)$ & $6.63(6.22-7.63)$ & $0.13(0.043-0.29)$ \\
$V_{E}$ children & $5.79(5.24-6.15)$ & $2.74(2.41-3.14)$ & $16.4(10.5-22.3)$ & $2.05(2.58-4.26)$ \\
$V_{\mathrm{A}}$ grandchildren & $10.4(7.86-14.5)$ & $2.63(1.31-3.87)$ & $73.1(65.1-78.9)$ & $2.28(0.61-4.39)$ \\
$V_{E}$ grandchildren & $55.7(51.9-59.8)$ & $9.12(7.97-10.7)$ & $11.3(9.16-13.3)$
\end{tabular}

Heritabilites, genetic variances $\left(V_{A}\right)$ and environmental variances $\left(V_{E}\right.$, all estimated after controlling for the variances due to maternal effects and birth cohort), for number of children and grandchildren born in two separate time periods before versus after the demographic transition and in two socioeconomic classes that correspond to different resource access within the predemographic transition subset. Heritabilities are estimated from z-standardised data, while genetic and environmental variances are estimated from models with mean-standardised variables.

tended to be higher in the landowning (0.92, CI: 0.86-0.96) subset than in the landless subset (0.79, CI: $0.36-0.93)$, indicating that number of children born tended to be least reliable as a predictor of long-term fitness in landless individuals before the demographic transition, and a more reliable predictor in landowning individuals.

\section{Robustness of results}

Both phenotypic and quantitative genetic models controlled for a range of fixed and random effects that have been shown to influence life history traits in this population (Methods section). Because individuals that do not reproduce per definition have both zero children and zero grandchildren, we tested if this influenced the results by running all models including only individuals that reproduced (individuals with one or more children and any number, including zero grandchildren). This did not qualitatively influence the results (details not shown). Results were also robust to a further division of landowners in the pre-industrial subset into 'wealthy' and 'average' landowners, resulting in three SES classes in total (details not shown). Quantitative genetic parameters did not differ significantly between the sexes (based on separate models in the two sexes, details not shown).

\section{DISCUSSION}

Our study utilises a large human data set, with our smallest subset comprising ca 1000 informative individuals. Despite this, a two- to threefold increase in sample size between subsets (Table 1) resulted in considerable increases in the precision of our estimates of genetic correlations. Nevertheless, the genetic correlation between number of children and grandchildren, while consistently high, was significantly $<1$ in all subsets (0.79-0.92), indicating that some of the variance in the genetic basis in number of grandchildren was independent of that in number of children. Thus, genetic variation in reproductive success does not entirely reflect the genetic variation in a longer-term estimate of fitness, number of grandchildren. This lends some support to previous findings of a quality/quantity trade-off in humans at the phenotypic level (Gillespie et al., 2008; Lawson and Mace, 2011; see also phenotypic correlations reported in the present study).

During industrialisation, mortality and death rates rapidly decreased. This likely lead to lessened quantity/quality trade-offs, at least in terms of child mortality risk, as most individuals had sufficient access to resources and smaller family sizes, allowing sufficient resource allocation to each offspring. Rapid changes in the environment can lead to changed trade-off patterns also on the genetic level. A growing number of empirical studies demonstrate that genetic variances and covariances between traits depend of the environment, which is not surprising because environment-specific allelic effects can change genetic covariances in a single generation (Wood and Brodie, 2015).
However, theoretical predictions regarding how genetic correlations respond to changes in environmental conditions are largely lacking (Arnold et al., 2008). Here, we performed the first test of how the genetic correlation between a short- and a long-term fitness measure depends on both differences in resource access between groups of individuals, and on rapid demographic changes over time. Overall, differences in the genetic correlations were small and non-significant (note the overlapping 95\% credibility intervals) and we therefore refrain from drawing strong conclusions regarding the patterns in the different subsets. Nonetheless, the slightly lower genetic correlation in the postindustrial period is in line with the prediction that life history traits should show a break-down of genetic correlations in novel environments (Sgrò and Hoffmann, 2004). Alternatively, some authors have suggested that modern environments may indeed serve to intensify relationships between parental investment and offspring success, triggering evolved mechanisms of fertility regulation to value offspring quality over quantity (Lawson and Mace, 2011). Taken together, our results indicate that number of grandchildren is a more accurate estimate of the genetic contribution to future generations than number of children, and is especially preferable in populations under resource restrictions. However, the gain in accuracy of predictions from using grandchildren rather than children has to be weighed against the added data requirements. The use of grandchildren requires complete life history data for three consecutive generations, which severely limits the sample size in most data sets.

Alternatively, shortcomings in the study design or study population selection could lead to a genetic correlation between numbers of children and grandchildren that is $<1$. Specifically, the results could be biased by the effects of inbreeding, measurement error and reverse causality, and we will go through these in turn. First, inbreeding in the population could diminish the estimates. However, although Finland has a decrease of genetic diversity compared with mainland Europe (Sajantila et al., 1996) due to recent founding bottlenecks (Jakkula et al., 2008), inbreeding is unlikely to have a major influence on our genetic estimates given that the pedigree-wide mean pairwise relatedness in our study was 0.00025 , and previous quantitative genetic estimates of life history traits from this and other Finnish historic data sets are in line with those found in other populations (Stearns et al., 2010). Second, measurement error in the number of children and grandchildren introduces noise and thus lowers the genetic correlation. We minimised this effect by only including individuals with complete recorded information on their own reproduction and the reproduction of all their children (when measuring number of grandchildren). Third, infant mortality can influence reproductive decisions and lead to increased fertility as an insurance or replacement strategy ('reversed causality'). However, number of grandchildren incorporates both offspring infant mortality (where reverse causality 
may indeed apply), as well as offspring adult mating success and reproductive output (where reverse causality does not apply). We therefore use an approach that is directly comparable to previous studies on this topic (for example, Zietsch et al., 2014).

A previous study on a post-demographic transition population in Sweden found a genetic correlation between number of children and grandchildren that was not significantly different from 1 (Zietsch et al., 2014). The population used in the present study comes from a country with similar culture and environment, yet despite this we found a genetic correlation that was significantly $<1$ under a comparable time period (0.88 after the demographic transition). Although this may nonetheless reflect differences between the populations, another reason for the discrepancy between the results may be related to the different methodologies that were applied. Twin and sibling designs are often used in studies on contemporary human populations. A critical assumption in analyses of twin studies is that monozygotic and dizygotic twins share a common environment. However, the validity of this assumption has been questioned (Devlin et al., 1997; Horwitz et al., 2003; Tropf et al., 2015). Further, maternal effects are rarely accounted for in twin designs, potentially leading to overestimation of additive genetic effects (Devlin et al., 1997). The twin design also excludes individuals from low fertility families, particularly only children, which may be especially problematic when studying the fertility of individuals (Tropf et al., 2015l). In addition, dizygotic twinning is heritable, meaning dizygotic twins potentially carry genes that are important for high fertility (Tropf et al., 2015). In historical populations, only limited data is available on twins. Thus, a statistical framework that utilises pedigree information to estimate additive genetic variances and covariances (the 'animal model') has been heavily used in evolutionary biology in recent decades (Henderson, 1975; Kruuk, 2004; Wilson et al., 2010) and has been successfully applied in numerous human studies (Pettay et al., 2005, 2008; Byars et al., 2010; Milot et al., 2011; Stearns et al., 2012; Bolund et al., 2013). Complex, natural pedigrees contain all levels of relatives (for example, siblings, grandparents and aunts/uncles as well as more distant relatives) which allows a separation of additive and non-additive genetic effects as well as the separation of genetic effects from cultural inheritance, at least to an appreciable degree. How successfully this is accomplished depends on the structure of the pedigree, because close relatives tend to also share a common environment. Estimates can therefore be inflated by shared environmental factors (Stearns et al., 2010). Thus, estimates of genetic variances and covariances are liable to be overestimated in both twin- and pedigree designs because the models capture both genetic and environmental variation when estimating the genetic component of a trait (Devlin et al., 1997; Horwitz et al., 2003; Keller et al., 2010; Stearns et al., 2010; Danchin et al., 2013). This should be taken into account when evaluating results of such studies, including ours, and means that the true genetic correlation between number of children and grandchildren is likely to be lower than estimated.

We found that both number of children and grandchildren were heritable, with heritabilities ranging from 10 to $16 \%$, with little differences depending on resource availability or time period. These heritability estimates are in line with previous findings from present and historical human populations (Kirk et al., 2001; Kohler et al., 2002; Pettay et al., 2005; Milot et al., 2011). Of more relevance to projections of evolutionary change is the actual amount of additive genetic variance, $V_{\mathrm{A}}$, and this showed clear differences depending on resource access level and time period. Gene expression depends on the environment (Lynch and Walsh, 1998) and $V_{\mathrm{A}}$ may be expected to increase under conditions of resource abundance (Hoffmann and Merilä, 1999) and in novel environments (Charmantier and Garant, 2005), potentially due to the release of cryptic genetic variation in novel or stressful environments, although the empirical evidence in support of this is limited (McGuigan and Sgrò, 2009; Wood and Brodie, 2015). As expected, landowners, with a more stable high resource access, expressed higher $V_{\mathrm{A}}$ than landless individuals before the demographic transition. However, contrary to this prediction, $V_{\mathrm{A}}$ decreased after the demographic transition. A previous study on a larger data set from the same population found that although the $V_{\mathrm{A}}$ of measures of timing of reproduction increased over the demographic transition, the $V_{\mathrm{A}}$ of number of children born did not change (Bolund et al., 2015). Alternatively, differences in $V_{\mathrm{A}}$ and $V_{\mathrm{E}}$ between subsets may reflect changes in phenotypic means and variances. Mirroring the patterns for $V_{\mathrm{A}}$ and $V_{\mathrm{E}}$ (Table 2), phenotypic means and variances of both number of children and grandchildren decreased over the demographic transition, and were higher in landowning than in landless individuals within the pre-transition subset (Table 1). Taken together, these findings demonstrate that the expressed additive genetic variance is sensitive to the measurement conditions and this needs to be considered in studies on different populations.

In conclusion, we found that patterns of resource availability need to be taken into account when estimating genetic covariances with fitness in human populations, otherwise life history trade-offs can be masked by variation in socioeconomic conditions. Further, studies that aim to predict evolutionary change over multiple generations should preferably use number of grandchildren rather than number of children as the fitness proxy, although the added data requirements needs to be taken into consideration. When number of grandchildren is not possible to estimate due to data limitation, LRS measures that account for some of the variation in offspring quality, such as survival to adulthood, may be preferable.

\section{DATA ARCHIVING}

The data analysed in this study concerns personal details of human subjects, some of whom are still alive. Therefore, access to the data set may be requested by contacting Prof. V Lummaa (virpi.lummaa@gmail.com).

\section{CONFLICT OF INTEREST}

The authors declare no conflict of interest.

\section{ACKNOWLEDGEMENTS}

We thank Lasse Iso-Iivari, Kimmo Pokkinen, Aino Siitonen, S Toijonen, J Piippo and the Karelian database for data collection. This work was funded by the Academy of Finland (VL) and the Wenner-Gren Foundations (EB).

Anderson KG (2006). How well does paternity confidence match actual paternity? Curr Anthropol 47: 513-520.

Arnold SJ, Burger R, Hohenlohe PA, Ajie BC, Jones AG (2008). Understanding the evolution and stability of the G-Matrix. Evolution 62: 2451-2461.

Bolund E, Bouwhuis S, Pettay JE, Lummaa V (2013). Divergent selection on, but no genetic conflict over, female and male timing and rate of reproduction in a human population. Proc Biol Sci 280: 20132002.

Bolund E, Hayward A, Lummaa V. Using quantitative genetics to understand life-history evolution in Humans. In the Encyclopedia of Evolutionary Biology, Kliman R, Coulson T (eds). Elsevier, Academic Press: Waltham, MA, USA, p 2132.

Bolund E, Hayward A, Pettay JE, Lummaa V (2015). Effects of the demographic transition on the genetic variances and covariances of human life-history traits. Evolution 69: 747-755.

Borgerhoff Mulder M (1998). The demographic transition: are we any closer to an evolutionary explanation? Trends Ecol Evol 13: 266-270. 
Brommer JE, Gustafsson L, Pietiäinen H, Merilä J (2004). Single-generation estimates of individual fitness as proxies for long-term genetic contribution. Am Nat 163: 505-517.

Byars SG, Ewbank D, Govindaraju DR, Stearns SC (2010). Natural selection in a contemporary human population. Proc Natl Acad Sci USA 107: 1787-1792.

Charmantier A, Garant D (2005). Environmental quality and evolutionary potential: lessons from wild populations. Proc Biol Sci 272: 1415-1425.

Charmantier A, Réale D (2005). How do misassigned paternities affect the estimation of heritability in the wild? Mol Ecol 14: 2839-2850.

Danchin E, Pujol B, Wagner RH (2013). The double pedigree: a method for studying culturally and genetically inherited behavior in tandem. PLos One 8: e61254.

De Jong G (1994). The fitness of fitness concepts and the description of natural selection. Q Rev Biol 69: 3-29.

Devlin B, Daniels M, Roeder K (1997). The heritability of IQ. Nature 388: 468-471.

Endler JA (1986). Natural Selection In The Wild. Princeton University Press: Princeton, NJ, USA.

Falconer DS, Mackay TFC (1996). Introduction To Quantitative Genetics. 4th edn. Prentice Hall: Harlow, UK.

Faurie C, Russell AF, Lummaa V (2009). Middleborns disadvantaged? Testing birth-order effects on fitness in pre-industrial Finns. PLos One 4: e5680.

Firth JA, Hadfield JD, Santure AW, Slate J, Sheldon BC (2015). The influence of nonrandom extra-pair paternity on heritability estimates derived from wild pedigrees. Evolution 69: 1336-1344.

Gillespie DOS, Russell AF, Lummaa V (2008). When fecundity does not equal fitness: evidence of an offspring quantity versus quality trade-off in pre-industrial humans. Proc Biol Sci 275: 713-722.

Goodman A, Koupil I, Lawson DW (2012). Low fertility increases descendant socioeconomic position but reduces long-term fitness in a modern post-industrial society. Proc R Soc Lond B Biol 279: 4342-4351.

Hadfield J (2010). MCMC methods for multi-response generalized linear mixed models: the MCMCgImm R package. J Stat Software 33: 1-22.

Hayward AD, Holopainen J, Pettay JE, Lummaa V (2012). Food and fitness: associations between crop yields and life-history traits in a longitudinally monitored pre-industrial human population. Proc Biol Sci 279: 4165-4173.

Henderson CR (1975). Best linear unbiased estimation and prediction under a selection model. Biometrics 31: 423-447.

Hoffmann AA, Merilä J (1999). Heritable variation and evolution under favourable and unfavourable conditions. Trends Ecol Evol 14: 96-101.

Horwitz AV, Videon TM, Schmitz MF, Davis D (2003). Rethinking twins and environments: possible social sources for assumed genetic influences in twin research. J Health Soc Behav 44: 111-129.

Hunt J, Bussiere LF, Jennions MD, Brooks R (2004). What is genetic quality? Trends Ecol Evol 19: 329-333

Jakkula E, Rehnström K, Varilo T, Pietiläinen OPH, Paunio T, Pedersen NL et al. (2008). The genome-wide patterns of variation expose significant substructure in a founder population. Am J Human Genet 83: 787-794.

Keller MC, Medland SE, Duncan LE (2010). Are extended twin family designs worth the trouble? A comparison of the bias, precision, and accuracy of parameters estimated in four twin family models. Behav Genet 40: 377-393.

Kirk KM, Blomberg SP, Duffy DL, Heath AC, Owens IPF, Martin NG (2001). Natural selection and quantitative genetics of life-history traits in Western women: a twin study. Evolution 55: 423-435.

Kohler HP, Rodgers JL, Christensen K (2002). Between nurture and nature: The shifting determinants of female fertility in Danish twin cohorts. Soc Bio/ 49: 218-248.

Kruuk LEB (2004). Estimating genetic parameters in natural populations using the 'animal model'. Philos Trans R Soc Lond B Biol Sci 359: 873-890.

Lack D (1947). The significance of clutch-size. IBIS 89: 302-352.

Larmuseau MHD, Vanoverbeke J, Van Geystelen A, Defraene G, Vanderheyden N, Matthys K et al. (2013). Low historical rates of cuckoldry in a Western European human population traced by Y-chromosome and genealogical data. Proc Biol Sci 280: 20132400.

Lawson DW, Mace R (2011). Parental investment and the optimization of human family size. Philos Trans $R$ Soc Lond B Biol Sci 366: 333-343.

Liu J, Lummaa V (2014). An evolutionary approach to change of status-fertility relationship in human fertility transition. Behav Ecol 25: 102-109.
Lummaa V, Haukioja E, Lemmetyinen R, Pikkola M (1998). Natural selection on human twinning. Nature 394: 533-534.

Lynch M, Walsh B (1998). Genetics And Analysis Of Quantitative Traits: Sinauer Associates: Sunderland, MA, USA.

McGraw JB, Caswell H (1996). Estimation of individual fitness from life-history data. Am Nat 147: 47-64.

McGuigan K, Sgrò CM (2009). Evolutionary consequences of cryptic genetic variation. Trends Ecol Evol 24: 305-311.

Meij JJ, Van Bodegom D, Ziem JB, Amankwa J, Polderman AM, Kirkwood TBL et al. (2009). Quality-quantity trade-off of human offspring under adverse environmental conditions. J Evol Biol 22: 1014-1023.

Milot E, Mayer FM, Nussey DH, Boisvert M, Pelletier F, Réale D (2011). Evidence for evolution in response to natural selection in a contemporary human population. Proc Natl Acad Sci USA 108: 17040-17045.

Pettay JE, Charmantier A, Wilson AJ, Lummaa V (2008). Age-specific genetic and maternal effects in fecundity of preindustrial Finnish women. Evolution 62: 2297-2304.

Pettay JE, Helle S, Jokela J, Lummaa V (2007). Natural selection on female life-history traits in relation to socio-economic class in pre-industrial Human populations. PLos One 2: e606.

Pettay JE, Kruuk LEB, Jokela J, Lummaa V (2005). Heritability and genetic constraints of life-history trait evolution in preindustrial humans. Proc Natl Acad Sci USA 102: 2838-2843.

Pinheiro J, Bates D, DebRoy S, Sarkar DeepayanR Development Core Team (2008).

Roff DA (2002). Life History Evolution. Sinauer Associates Inc.: MA, USA.

Sajantila A, Salem AH, Savolainen P, Bauer K, Gierig C, Pääbo S (1996). Paternal and maternal DNA lineages reveal a bottleneck in the founding of the Finnish population. Proc Natl Acad Sci USA 93: 12035-12039.

Schielzeth H (2010). Simple means to improve the interpretability of regression coefficients. Methods Ecol Evol 1: 103-113.

Sgrò CM, Hoffmann AA (2004). Genetic correlations, tradeoffs and environmental variation. Heredity 93: 241-248

Singleton F (1998). A Short History of Finland2 edn.Cambridge University Press: Cambridge, UK.

Skjærvø GR, Bongard T, Viken Å, Stokke BG, Røskaft E (2011). Wealth, status, and fitness: a historical study of Norwegians in variable environments. Evol Hum Behav 32: 305-314

Stearns SC (1976). Life-history tactics: a review of the ideas. Q Rev Biol 51: 3-47.

Stearns SC, Byars SG, Govindaraju DR, Ewbank D (2010). Measuring selection in contemporary human populations. Nat Rev Genet 11: 611-622.

Stearns SC, Govindaraju DR, Ewbank D, Byars SG (2012). Constraints on the coevolution of contemporary human males and females. Proc Biol Sci 279: 4836-4844.

Sundin J (1992). Sinful sex - Legal prosecution of extramarital sex in preindustrial Sweden. Soc Sci Hist 16: 99-128.

Tropf FC, Stulp G, Barban N, Visscher PM, Yang J, Snieder H et al. (2015). Human fertility, molecular genetics, and natural selection in modern societies. PLos One 10: e0126821.

van Noordwijk AJ, Jong Gd (1986). Acquisition and allocation of resources: their influence on variation in life history tactics. Am Nat 128: 137-142.

Venables WN, Ripley BD (2002). Modern Applied Statistics with SFourth edn.Springer: New York, NY, USA.

Voland E (1990). Differential reproductive success within the Krummhörn population (Germany, 18th and 19th centuries). Behav Ecol Sociobiol 26: 65-72.

Wells JCK, Stock JT (2007). The biology of the colonizing ape. Am J Phys Anthropol 134: 191-222.

Wilson AJ, Réale D, Clements MN, Morrissey MM, Postma E, Walling CA et al. (2010). An ecologist's guide to the animal model. J Anim Ecol 79: 13-26.

Wolf JB, Wade MJ (2001). On the assignment of fitness to parents and offspring: whose fitness is it and when does it matter? J Evol Biol 14: 347-356.

Wood CW, Brodie ED (2015). Environmental effects on the structure of the G-matrix. Evolution 69: 2927-2940.

Zietsch BP, Kuja-Halkola R, Walum H, Verweij KJH (2014). Perfect genetic correlation between number of offspring and grandoffspring in an industrialized human population. Proc Natl Acad Sci USA 111: 1032-1036.

Supplementary Information accompanies this paper on Heredity website (http://www.nature.com/hdy) 